\title{
Immunopharmacogenomics in Cancer Management
}

\author{
Gizem Calibasi-Kocal and Yasemin Baskin \\ Additional information is available at the end of the chapter \\ http://dx.doi.org/10.5772/intechopen.76934
}

\begin{abstract}
With the unavoidable progress of genomics technologies, "one size fits all" strategy has switched to individual-specific treatment approaches. Hence pharmacogenomics-based personalized cancer medicine has emerged. Promising treatment option immunotherapy includes either "take the brakes off immune system (i.e., checkpoint blockade therapy) or the use of immune cells expanded in an in vitro tumor-free environment". Both options have been varied and included unpredictable results. Combination of cancer immunotherapy and pharmacogenomics applications may contribute to solve the complexity of outcome prediction and variations between individuals receiving the same immunotherapeutic treatment. To enhance the tumor immunity and determine cancer patients who response to immunotherapy, classification based on gene polymorphisms in key immunoregulatory molecules including antigen-presenting molecules, immunoglobulins and their receptors, cytokine/chemokines and their receptors, adhesion and costimulatory molecules, toll-like receptors, and intracellular signaling molecules plays a vital role in redirecting or modulating the function of immune cells. Therefore, polymorphisms in immunoregulatory molecules and their impact on immunotherapeutic outcome should be considered in cancer management.
\end{abstract}

Keywords: cancer, immunopharmacogenomics, personalized immunotherapy, polymorphisms

\section{Introduction}

The host immune system has a crucial role on the pathogenesis of many diseases, including cancer. Tumorigenesis depends not only on cellular biology but also on immune responses. The tumor development and progression are associated with the interaction between the immune system and malignant cells. The effect of immune responses has been counted on for both cancer immunotherapies and a broad range of cytotoxic agents as well as molecular 
targeted drugs. With the accumulation of information about the immune responses in tumor immune surveillance, the fame of cancer immunotherapy is increasing. Some cancer immunotherapies, such as monoclonal antibodies, cytokines, cancer vaccines, and cell-based therapies, have been developed and incorporated into clinical practice for the activation of the host immune response to eliminate cancer cells [1, 2].

\section{Importance of personalized cancer therapy on immunotherapy}

With characterization of alterations on immunity-related molecules through deep sequencing tools in the genomic era, immunotherapies are revolutionizing cancer treatment. Natural variations in nucleic acid sequence named as gene polymorphisms exist in general population with a high frequency and include no side effect for the people. Single nucleotide polymorphisms (SNPs) are the most common polymorphism in population [3]. SNPs can be also recognized as biological markers to identify genes that are associated with diseases. It has been displayed that some SNPs have effects on individual's response to some drugs. Therefore research groups are focused on the investigation of how SNPs in the human genome correlate with drug response to get more successful therapy regimes in personalized medicine era [4]. At this point, immunopharmacogenomics that incorporates immunogenomics and pharmacogenomics aims to improve a better approach for how the immune system impacts to the immunotherapy response.

\subsection{PD-1/PD-L1 polymorphisms}

The blockade of immune checkpoints modulating immune responses has recently been emerged as an immune therapy against cancer. Different immune checkpoint molecules such as programmed cell death 1 receptor (PD-1), an immune checkpoint receptor on immune cells (especially on T lymphocytes); and programmed death-1 ligand-1 (PD-L1) on tumor cells or tumor-infiltrating immune cells, have been associated with tumor immune evasion [5]. Interaction of PD-1 with PD-L1 starts antitumor immune response suppression; therefore blockade of PD-1/PD-L1 binding has been recently applied for antitumor immune therapy [6]. PD-1 is expressed on some immune cells, including T cells, natural killer T (NKT) cells, mature $\mathrm{CD}^{+}$and $\mathrm{CD}^{+} \mathrm{T}$ cells, some dendritic cells, B cells, lymph node, and bone marrow cells [4]. PD-1 is commonly expressed immunoinhibitory receptor that belongs to CD28/B7 family, and it is expressed on some immune cells, including T cells, natural killer T (NKT) cells, mature $\mathrm{CD}^{+}$and $\mathrm{CD}^{+} \mathrm{T}$ cells, some dendritic cells, B cells, lymph node, and bone marrow cells. PD-1 has two opposite roles such as downregulation of ineffective immune responses and expansion of malignant cells by preventing of protective antitumor immune responses [7]. PD-1 has function on the inhibition of T-cell activation, production, and survival [4]. PD-L1, ligand of PD-1, is a member of the B7 family of immune-regulatory ligands. It is expressed on functioning active T cells, B cells, dendritic cells, and macrophages (antigen-presenting cells) and activates various tissue groups by inflammatory cytokines [7]. PD-L1 has two different forms as membrane bound and soluble form. Both of these forms have been found on CD28/ B27 family such as CTLA-4, CD28, and B7-H4 [8]. 
PD-L1 has role on the negative regulation of immunological response. PD-L1 overexpression can prevent to form antitumor immune responses against cancer cells; hence increased expression of PD-L1 on tumor cells may be predictive for a blockade of the PD-L1/PD-1 binding [9]. Some studies have shown that PD-L1 is also expressed on many types of cancer cells (including melanoma, lung cancer), and it has been reported that expression of PD-L1 ligands by cancer cells results in the evasion of the immune system. They stop the production of tumor-specific T cells by propagation of inhibitory signals which cause damaged antitumor immunity $[7,10]$. Based on their function on antitumor immune response suppression, PD-1 and PD-L1 can be considered as powerful biomarkers for new tumor formation or progression of cancer. In literature some studies are focused on their role on genetic susceptibility, and some of them are focused on their prognostic or predictive significance. In literature some polymorphisms on PD-1 which is encoded by PDCD-1 gene (Gene bank ID: 5153) on chromosome 2q37 location are associated with tumor susceptibility. $P D-1.1$ polymorphism (dbSNP reference cluster ID rs36084323, c.-606G > A), located in the promoter region, has been accepted as a risk factor for non-small cell lung cancer and breast cancer [11, 12]. PD-1.3 (rs11568821, c.627 + 189G > C, ) located in intron 4 has been associated with colon, thyroid, and breast cancer [13-15]. PD-1.5 (rs2227981, c.804 T > C), located in exon 5, is associated with increased cancer risks in nonsmall cell lung, cervical, colon, and ovarian cancer $[4,16]$. A recent study showed that $P D-1.9$ (rs2227982, c.644C > T), located in exon 5, has significance on the gastric cancers [17]. PD-L1 gene (Gene bank ID: 29126) polymorphisms (rs2890658, c.683-369C > A, and rs10815225, c.-114C > A) might serve as risk markers for esophageal squamous cell carcinoma and gastric cancer [18, 19]. Blockade of PD-1/PD-L1 pathway with specific anti-PD-1 treatments such as nivolumab, lambrolizumab, pidilizumab, BMS-93659 (MDX-1105), RG7446/MPDL3280, and MEDI4736 (B7H1) has been tested in different cancers including ovarian, colorectal, bladder, and non-small cell lung cancer and melanoma. Even though responses vary in different tumor types, antiPD-1 treatments are supported. Responses not only vary in different tumor types; some patients show clear responses to blockade of PD-1/PD-L1 pathway, whereas they do not respond [4]. Currently, it is difficult to predict treatment responses to anti-PD-1 treatments. Expression of PD-L1 is not an optimal biomarker, because determination of PD-L1 expression by immunohistochemistry is not always proper due to the different antibody selection and cutoff levels. The PD-L1 expression may not anticipate the therapeutic responses because the PD-1/PD-L1 interactions on B cells have role on adaptive and innate immune systems. Nowadays, researchers are focused on the potential impact of single nucleotide polymorphisms as predictive therapeutic markers and their improvement capacity of the expression markers [20]. In literature, there are some studies about the effect of $P D-1 / P D-L 1$ SNPs on non-small-cell lung cancer (NSCLC) treatment [20-22]. Nomizo et al. evaluated the association between PD-1/PD-L1 SNPs and response to nivolumab, PD-1 immune checkpoint inhibitor, and survival in NSCLC. Five SNPs in PD-L1 (rs1411262, c.394 + 1999C > A; rs2282055; c.-14-368 > G; rs4143815; c.*395G > C; rs2890658;c.683$369 \mathrm{C}>\mathrm{A}$; rs822339, c. $-15+2576 \mathrm{~A}>\mathrm{G}$ ) and two SNPs in PD-1 (rs2227981; c.804 T > C; rs2227982, c.644C > T) were investigated by using real-time polymerase chain reaction (RT-PCR). The G allele of rs2282055 and the C allele of rs4143815 in PD-L1 were significantly associated with better clinical response than T or G allele, respectively [20]. In a study conducted by Do et al., SNPs involved in immune checkpoints were used to predict the clinical outcomes of patients with advanced-stage NSCLC after paclitaxel-cisplatin chemotherapy. The SNP rs2297136, c. ${ }^{* 93 G}>$ A 
in PD-L1 gene, was significantly associated with better chemotherapy response and better overall survival, and rs4143815 in PD-L1 was also found as associated with better response to paclitaxel-cisplatin chemotherapy [21]. $P D$-L1 polymorphisms, especially rs4143815, may be promising markers for the prediction of clinical outcome of chemotherapy. However further studies with larger patient groups are needed to validate the results from different research groups, and also more studies should be designed to figure out PD-L1 in chemotherapy responses of different cancers.

\subsection{CTLA4 polymorphisms}

After the determination and characterization of cytotoxic T-lymphocyte-associated protein 4 (CTLA-4) as a key negative regulator in immune response (broadly named as checkpoint molecule), studies have increased to develop cancer immunotherapy targeting this co-inhibitory molecule. CTLA-4 (CD152) is a member of immunoglobulin (Ig) superfamily and functions for downregulation of T-cell activation. CTLA-4 expressed by activated T cells binds to B7.1 (CD80) and B7.2 (CD86) on antigen-presenting cells and transmits an inhibitory signal to T cells, so it can restrict the density and extent of the immune responses. The complex formed from CTLA-4 and B7 proteins (B7.1 and B7.2) can switch activated T cells into inhibitory T cells. This change promotes tumor escape from immunosurveillance [23]. Although lymphoproliferative disorders and severe autoimmune diseases have been shown in CTLA4-knockout mice; CTLA- 4 blockade can boost immune responses in tumor-transplanted mice, as well as extending antitumor immune responses and rejection of tumors [24, 25]. It has been shown that blocking of CTLA-4, namely, downregulation of T-cell activation, can lead the cancer regression in patients with different cancers due to the enhancing immune responses as well as antitumor activity $[23,26]$. However, not all patients can benefit from the treatment of CTLA-4 blockade, and some of them developed severe autoimmune reactions. The mechanisms of this interindividual variability in response to immunotherapy are not well understood.

Some CTLA-4 polymorphisms have been investigated with the determination of genetic factors that may change the response and toxicity of CTLA- 4 antibody therapy [27]. Human CTLA-4 gene with four exons is located on chromosome $2 q 33$, and different functional subunits of CTLA-4 protein such as a leader sequence, an extracellular, a transmembrane, and a cytoplasmic domain are encoding [28]. Many SNPs have been identified in the CTLA-4 gene that contains three exons and two introns. To date, at least five SNPs that were well studied in immune diseases have been investigated in cancer association studies. Among them, the rs5742909, c. $-318 \mathrm{C}>\mathrm{T}$ located in the promoter region; rs231775, c.49A > G located in exon 1; and rs3087243, c.6230A > G (CT60) located in 3'-untranslated region (3'UTR) have attracted more attention. There are more than 100 SNPs in CTLA-4 gene, and most of them are spotted on three exons and two introns of CTLA-4 gene. Until now, at least five attracted SNPs have been associated in cancer-related studies, such as rs11571316, c. - 1577G > A in the 5'-untranslated region (5'UTR); rs5742909, c. $-318 \mathrm{C}>\mathrm{T}$ in the promoter region; rs231775, c.49A > G in exon 1; and rs3087243, c.6230A > G (CT60) in 3'-untranslated region (3'UTR). These alterations change the expression or functional activity of CTLA-4 protein [29]. Some common SNPs have been reported that they alter either expression or function of the CTLA-4 gene product, as well as responses to immunotherapy with anti-CTLA-4 antibodies. A study conducted in melanoma 
by Breunis et al. has shown that $\mathrm{G}$ allele of rs4553808, c.-1660A > G; T allele of rs11571317, c. $-657 \mathrm{C}>\mathrm{T}$; and A allele rs231775, c.49A > G, are significantly associated with response to antiCTLA-4 antibody (MDX-010). SNPs rs4553808 and rs11571317 are spotted on the promoter region; and rs231775 is on the exon 1 and causes p.Thr17Ala substitution [27]. In another study performed by Queirolo P. et al. in metastatic melanoma patients, rs11571316, c. - 1577G > A in the 5'UTR, and rs3087243, c.6230A > G (CT60) in the 3'UTR of the gene, were associated with best overall response to ipilimumab which is a CTLA-4 immune checkpoint blocker [30]. In lung cancer-based study, which is performed by Song B et al., patients with the A allele had a significantly shorter survival time than those with the $G$ allele ( $p$ value $<0.001$; survival times: AA genotype 9.8 months, GA genotype 12.0 months, GG genotype 12.5 months) [31]. With the presented studies above, it can be said that several SNPs in CTLA-4 are biologically functional and might be predictive factors for CTLA-4 antibody therapy used for cancers.

\subsection{CCR5 polymorphisms}

Pro-inflammatory cytokines, chemokines and other protein-structured molecules are secreting from cancerous cells and their stromal environment. Chemokine family belongs to chemotactic cytokines and has 44 members. Chemokines have role in homeostasis and immunity by controlling the leukocyte trafficking and induction, so they have role in organogenesis, cell growth, and differentiation. Tumor-related chemokines have important role in cancer biology, in terms of leukocyte infiltration, angiogenesis, and immune evasion. Based on the number and spacing of conserved cysteine residues on their N-terminus, chemokines consist of four subgroups named as CX3C, CXC, CC, and C [32,33]. Chemokines act through their interactions with specific chemokine receptors located on the cell surface. Most of the cancerous cells overexpress these specific chemokine receptors which have role together with chemokines in cellular survival, proliferation, migration, invasion, and metastasis. In humans, the chemokine receptor family includes 24 members, and they are divided into four subfamilies (CX3CR, CXCR, CCR, and CR) based on the class of chemokines that they interact. Specific ligand-receptor binding induces GDP to GTP exchange, and this activates the downstream signaling effectors for the propagation of cellular pathways [34, 35].

It is considered that chemokine receptors are promising targets for new immunotherapies since the detection of overexpressed chemokine receptors on the surface of cancer cells [36]. Systemic administration of interleukin-2 and checkpoint inhibitors increases the secretion of chemoattractants such as ligands of CCR5 and CXCR. Therefore polymorphisms on CCR5 and CXCR chemokine receptors affect the migration capability of tumor-infiltrating lymphocytes to the tumor location [37]. So response rates to immunotherapy vary among patients. Ugural et al. showed that genotypic status of CCR5 chemokine receptor has an impact on the immunotherapy (interferons or interleukin-2) responses in patients with metastatic melanoma. A strong association was determined between the CCR5 $\Delta 32$ genotype and overall survival as poor prognostic factor. CCR5 $\Delta 32$ polymorphism (rs333, c.554-585del32) consists of 32 bp deletion. Heterozygosity causes decreased levels of receptor expression, and homozygosity causes the absence of expression. Immunotherapy outcome was worse in metastatic melanoma patients who had CCR5 332 genotype as homozygotes or heterozygotes [38]. Above observation of Ugurel et al. clashes with findings of study performed by Bedognetti et al. 
The presence of CCR5 32 polymorphism was not correlated with worse response in metastatic melanoma patients undergoing adoptive therapy [39]. Conflicting studies exist on the effect of CCR5 332 polymorphism on immunotherapy or immunochemotherapy. Hamid et al. did not report any significant relationship between the existence of CCR5 332 genotype or rs1799987, c.-301 + 246A > G, polymorphism and responsiveness to ipilimumab [40].

\subsection{KIR, HLA, and Fc $\gamma$ polymorphisms}

Natural killer (NK) cells, large granular lymphocytes, have role on the early innate immune response. In contrast to $\mathrm{T}$ cells, which remember foreign antigens through T-cell receptors in the context of major histocompatibility complex (MHC) molecules, NK cells are programmed to eliminate infected or transformed cells. Activation of NK cells, known as "missing-self" model due to the absence of MHC molecules depends on the numerous signals through their respective activating or inhibitory receptors [41]. The killer immunoglobulin-like receptor (KIR) gene encodes both activating and inhibitory NK-cell surface receptors. HLA class I gene encodes ligands of inhibitory KIR (HLA-C1 for KIR2DL2/3, HLA-C2 for KIR2DL1, HLABw4 for KIR3DL1), and the interaction between KIR and HLA class I ligands leads to NK inhibition. NK cells also express Fc receptors [42]. The interplay between KIR and HLA is crucial for positive outcomes of immune-related therapies. KIR/KIR-ligand mismatch is associated with improved outcome to immune-related therapies as well as autologous stem cell transplantation [42].

Delgado et al. reported that KIR receptor-ligand mismatch was related with response or improvement of relapsed or refractory neuroblastoma patients receiving interleukin-2-based treatment, consistent with a role for NK cells in this clinical response [43].

There are three fragment $c$ gamma receptor $(\mathrm{Fc} \gamma \mathrm{R})$ classes such as (i) FcgRI, capable of highaffinity binding monomeric IgG, (ii) FcgRII with low-affinity binding, and (iii) FcgRIII interaction with complexed IgG. FcgRII and FcgRIII have variants with different binding affinity immune complexes such as FcgRIIa (131H/R) and FcgRIIIa (Val158Phe) [44].

Several groups have investigated the role of $F c \gamma R$ polymorphisms in the response of monoclonal antibodies (mAbs) such as rituximab, cetuximab, and trastuzumab. A correlation was reported between FcgRIIa polymorphisms and complete response in rituximab-based regimen received by non-Hodgkin lymphoma patients [44]. In another study performed to understand the influence of the FCGR3A gene polymorphism on rituximab response of nonHodgkin lymphoma patients, again a correlation was found between FCGR3A genotype and clinical response [45]. In a preliminary study, FCGR2A and FCGR3A polymorphisms were shown as useful markers to predict clinical outcome in metastatic colorectal cancer patients treated with cetuximab, a chimeric immunoglobulin G1 (IgG1) monoclonal antibody (mAb) against epidermal growth factor receptor (EGFR) [46]. Musolino et al. reported a correlation between FCGR3A and objective response rate and progression-free survival in patients with HER-2/neu-amplified breast cancer receiving trastuzumab. Also an association was determined between the combination of FCGR2A and FCGR3A and better objective response rate and progression-free survival [47]. Cheung et al. reported an association between $F c \gamma R 2 A$ polymorphism and progression-free survival in the response of neuroblastoma patients to the anti-GD2 antibody [48]. As a result, KIR-HLA immunogenetics can give useful information 
about the activation of innate immunity, and combining KIR-HLA genotyping with other molecular markers such as $\mathrm{F}_{\mathrm{C}} \gamma$ receptor polymorphisms that can give insight about immune responsiveness may allow clinicians to determine the most effective and least toxic personalized immunotherapy.

\section{Conclusion and future aspects}

Immunogenomics uses advance genomic analysis tools to distinguish the limitations of the immune system, and pharmacogenomics identifies the variability of pharmacologic responses based on individual's genetic/germline variations. Integration of both immunogenomics and pharmacogenomics forms immunopharmacogenomics to revolutionize the immunotherapy applications through the identification of genetic status of immunoregulatory molecules. This approach could be used to develop a better understanding for immunologic reactions, select patients for immunotherapy, and predict the side effects and response to anticancer treatment (not only immunotherapy but also chemo/radiation therapy). Although the immunopharmacogenetic applications are limited in clinical practice, it is clear that immunopharmacogenomics will become an important approach of cancer management in immunotherapy era.

\section{Conflict of interest}

Authors declare no conflict of interest.

\section{Author details}

Gizem Calibasi-Kocal ${ }^{1}$ and Yasemin Baskin ${ }^{2,3 *}$

*Address all correspondence to: yasemin.baskin@deu.edu.tr

1 Department of Basic Oncology, Institute of Oncology, Dokuz Eylul University, Izmir, Turkey

2 Department of Biostatistics and Medical Informatics, Faculty of Medicine, Dokuz Eylul University, Izmir, Turkey

3 Personalized Medicine and Pharmacogenomics Research Center, Dokuz Eylul University, Izmir, Turkey

\section{References}

[1] Farkona S, Diamandis EP, Blasutig IM. Cancer immunotherapy: The beginning of the end of cancer? BMC Medicine. 2016;14:73. DOI: 10.1186/s12916-016-0623-5 
[2] Palucka AK, Coussens LM. The basis of oncoimmunology. Cell. 2016;164(6):1233-1247. DOI: 10.1016/j.cell.2016.01.049

[3] Snyder MW, Adey A, Kitzman JO, Shendure J. Haplotype-resolved genome sequencing: Experimental methods and applications. Nature Reviews. Genetics. 2015;16(6):344-358. DOI: $10.1038 / \mathrm{nrg} 3903$

[4] Salmaninejad A, Khoramshahi V, Azani A, Soltaninejad E, Aslani S, Zamani MR, Zal M, Nesaei A, Hosseini SM. PD-1 and cancer: Molecular mechanisms and polymorphisms. Immunogenetics. 2018;70(2):73-86. DOI: 10.1007/s00251-017-1015-5

[5] Marin-Acevedo JA, Soyano AE, Dholaria B, Knutson KL, Lou Y. Cancer immunotherapy beyond immune checkpoint inhibitors. Journal of Hematology \& Oncology. 2018;11(1):8. DOI: 10.1186/s13045-017-0552-6

[6] Xu-Monette ZY, Zhang M, Li J, Young KH. PD-1/PD-L1 Blockade: Have we found the key to unleash the antitumor immune response? Frontiers in Immunology. 2017;8:1597. DOI: 10.3389/fimmu.2017.01597

[7] Gianchecchi E, Delfino DV, Fierabracci A. Recent insights into the role of the PD-1/PD-L1 pathway in immunological tolerance and autoimmunity. Autoimmunity Reviews. 2013;12(11):1091-1100. DOI: 10.1016/j.autrev.2013.05.003

[8] Cheng S, Zheng J, Zhu J, Xie C, Zhang X, Han X, Song B, Ma Y, Liu J. PD-L1 gene polymorphism and high level of plasma soluble PD-L1 protein may be associated with non-small cell lung cancer. The International Journal of Biological Markers. 2015;30(4):e364-e368. DOI: $10.5301 / \mathrm{jbm} .5000170$

[9] Yeo MK, Choi SY, Seong IO, Suh KS, Kim JM, Kim KH. Association of PD-L1 expression and PD-L1 gene polymorphism with poor prognosis in lung adenocarcinoma and squamous cell carcinoma. Human Pathology. 2017;68:103-111. DOI: 10.1016/j. humpath.2017.08.016

[10] Velcheti V, Schalper KA, Carvajal DE, Anagnostou VK, Syrigos KN, Sznol M, Herbst RS, Gettinger SN, Chen L, Rimm DL. Programmed death ligand-1 expression in nonsmall cell lung cancer. Laboratory Investigation. 2014;94(1):107-116. DOI: 10.1038/ labinvest.2013.130

[11] Sasaki H, Tatemaysu T, Okuda K, Moriyama S, Yano M, Fujii Y. PD-1 gene promoter polymorphisms correlate with a poor prognosis in non-small cell lung cancer. Molecular and Clinical Oncology. 2014;2(6):1035-1042. DOI: 10.3892/mco.2014.358

[12] Hua Z, Li D, Xiang G, Xu F, Jie G, Fu Z, Jie Z, Da P, Li D. PD-1 polymorphisms are associated with sporadic breast cancer in Chinese Han population of Northeast China. Breast Cancer Research and Treatment. 2011;129(1):195-201. DOI: 10.1007/s10549-011-1440-3

[13] Yousefi AR, Karimi MH, ShamsdinSA, Mehrabani D, Hosseini SV, Erfani N, Bolandparvaz S, Bagheri K. PD-1 gene polymorphisms in Iranian patients with colorectal cancer. Lab Medicine. 2013;44:241-244. DOI: 10.1309/LMG1BS4J3TAONRQF 
[14] Haghshenas MR, Dabbaghmanesh MH, Miri A, Ghaderi A, Erfani N. Association of PDCD1 gene markers with susceptibility to thyroid cancer. Journal of Endocrinological Investigation. 2017;40(5):481-486. DOI: 10.1007/s40618-016-0579-5

[15] Haghshenas MR, Naeimi S, Talei A, Ghaderi A, Erfani N. Program death 1 (PD1) haplotyping in patients with breast carcinoma. Molecular Biology Reports. 2011;38(6):4205-4210. DOI: 10.1007/s11033-010-0542-Z

[16] Yin L, Guo H, Zhao L, Wang J. The programmed death-1 gene polymorphism (PD-1.5 $\mathrm{C} / \mathrm{T}$ ) is associated with non-small cell lung cancer risk in a Chinese Han population. International Journal of Clinical and Experimental Medicine. 2014;7(12):5832-5836

[17] Zhou RM, Li Y, Wang N, Huang X, Cao SR, Shan BE. Association of programmed death-1 polymorphisms with the risk and prognosis of esophageal squamous cell carcinoma. Cancer Genetics. 2016;209(9):365-375. DOI: 10.1016/j.cancergen.2016.06.006

[18] Zhou RM, Li Y, Liu JH, Wang N, Huang X, Cao SR, Shan BE. Programmed death-1 ligand-1 gene rs2890658 polymorphism associated with the risk of esophageal squamous cell carcinoma in smokers. Cancer Biomarkers. 2017;21(1):65-71. DOI: 10.3233/ CBM-170269

[19] Tao LH, Zhou XR, Li FC, Chen Q, Meng FY, Mao Y, Li R, Hua D, Zhang HJ, Wang WP, Chen WC. A polymorphism in the promoter region of PD-L1 serves as a bindingsite for SP1 and is associated with PD-L1 overexpression and increased occurrence of gastric cancer. Cancer Immunology, Immunotherapy. 2017;66(3):309-318. DOI: 10.1007/ s00262-016-1936-0

[20] Nomizo T, Ozasa H, Tsuji T, Funazo T, Yasuda Y, Yoshida H, Yagi Y, Sakamori Y, Nagai H, Hirai T, Kim YH. Clinical impact of single nucleotide polymorphism in PD-L1 on response to nivolumab for advanced non-small-cell lung cancer patients. Scientific Reports. 2017;7:45124. DOI: 10.1038/srep45124

[21] Do SK, Lee SY, Choi JE, Hong MJ, Lee JH, Park JY. PD-L1 polymorphism can predict clinical outcomes of non-small cell lung cancer patients treated with first-line paclitaxelcisplatin chemotherapy. Journal of Thoracic Oncology. 2016;11(4 Suppl):S125. DOI: 10.1038/srep25952

[22] Lee SY, Jung DK, Choi JE, Jin CC, Hong MJ, Do SK, Kang HG, Lee WK, Seok Y, Lee EB, Jeong JY, Shin KM, Yoo SS, Lee J, Cha SI, Kim CH, Park JY. Functional polymorphisms in PD-L1 gene are associated with the prognosis of patients with early stage non-small cell lung cancer. Gene. 2017;599:28-35. DOI: 10.1016/j.gene.2016.11.007

[23] Hodi FS, Mihm MC, Soiffer RJ, Haluska FG, Butler M, Seiden MV, Davis T, HenrySpires R, MacRae S, Willman A, Padera R, Jaklitsch MT, Shankar S, Chen TC, Korman A, Allison JP, Dranoff G. Biologic activity of cytotoxic T lymphocyte-associated antigen 4 antibody blockade in previously vaccinated metastatic melanoma and ovarian carcinoma patients. Proceedings of the National Academy of Sciences of the United States of America. 2003;100(8):4712-4717. DOI: 10.1073/pnas.0830997100 
[24] Sun T, Hu Z, Shen H, Lin D. Genetic polymorphisms in cytotoxic T-lymphocyte antigen 4 and cancer: the dialectical nature of subtle human immune dysregulation. Cancer Research. 2009;69(15):6011-6014. DOI: 10.1158/0008-5472.CAN-09-0176

[25] Ma Y, Liu X, Zhu J, Li W, Guo L, Han X, Song B, Cheng S, Jie L. Polymorphisms of coinhibitory molecules (CTLA-4/PD-1/PD-L1) and the risk of non-small cell lung cancer in a Chinese population. International Journal of Clinical and Experimental Medicine. 2015;8(9):16585-16591

[26] Phan GQ, Yang JC, Sherry RM, Hwu P, Topalian SL, Schwartzentruber DJ, Restifo NP, Haworth LR, Seipp CA, Freezer LJ, Morton KE, Mavroukakis SA, Duray PH, Steinberg SM, Allison JP, Davis TA, Rosenberg SA. Cancer regression and autoimmunity induced by cytotoxic $\mathrm{T}$ lymphocyte-associated antigen 4 blockade in patients with metastatic melanoma. Proceedings of the National Academy of Sciences of the United States of America. 2003;100(14):8372-8377. DOI: 10.1073/pnas.1533209100

[27] Breunis WB, Tarazona-Santos E, Chen R, Kiley M, Rosenberg SA, Chanock SJ. Influence of cytotoxic $\mathrm{T}$ lymphocyte-associated antigen 4 (CTLA4) common polymorphisms on outcome in treatment of melanoma patients with CTLA-4 blockade. Journal of Immunotherapy. 2008;31(6):586-590. DOI: 10.1097/CJI.0b013e31817fd8f3

[28] Qi P, Ruan CP, Wang H, Zhou FG, Xu XY, Gu X, Zhao YP, Dou TH, Gao CF. CTLA-4 $+49 \mathrm{~A}>\mathrm{G}$ polymorphism is associated with the risk but not with the progression of colorectal cancer in Chinese. International Journal of Colorectal Disease. 2010;25(1):3945. DOI: $10.1007 / \mathrm{s} 00384-009-0806-\mathrm{z}$

[29] Xiaolei L, Baohong Y, Haipeng R, Shuzhen L, Jianfeng G, Xiangpo P, Haiyu L, Yuan Y, Dejie Z, Jinhong Y, Huanxin W, Wenhui W, Guohua Y. Current evidence on the cytotoxic T-lymphocyte antigen $4+49 \mathrm{G}>\mathrm{A}$ polymorphism and digestive system cancer risks: A meta-analysis involving 11,923 subjects. Meta Gene. 2015;6:105-108. DOI: 10.1016/j. mgene.2015.09.005

[30] Queirolo P, Dozin B, Morabito A, Banelli B, Piccioli P, Fava C, Leo C, Carosio R, Laurent S, Fontana V, Ferrucci PF, Martinoli C, Cocorocchio E, Battaglia A, Ascierto PA, Capone M, Simeone E, De Galitiis F, Pagani E, Antonini Cappellini GC, Marchetti P, Guida M, Tommasi S, Mandalà M, Merelli B, Quaglino P, Fava P, Guidoboni M, Romani M, Spagnolo F, Pistillo MP. Association of CTLA-4 gene variants with response to therapy and long-term survival in metastatic melanoma patients treated with ipilimumab: An Italian Melanoma Intergroup Study. Frontiers in Immunology. 2017;8:386. DOI: 10.3389/ fimmu.2017.00386

[31] Song B, Liu Y, Liu J, Song X, Wang Z, Wang M, Zhu Y, Han J. CTLA-4 +49A>G polymorphism is associated with advanced non-small cell lung cancer prognosis. Respiration. 2011;82(5):439-444. DOI: 10.1159/000329345

[32] Vela M, Aris M, Llorente M, Garcia-Sanz JA, Kremer L. Chemokine receptor-specific antibodies in cancer immunotherapy: Achievements and challenges. Frontiers in Immunology. 2015;6:12. DOI: 10.3389/fimmu.2015.00012 
[33] Bachelerie F, Ben-Baruch A, Burkhardt AM, Combadiere C, Farber JM, Graham GJ, Horuk R, Sparre-Ulrich AH, Locati M, Luster AD, Mantovani A, Matsushima K, Murphy PM, Nibbs R, Nomiyama H, Power CA, Proudfoot AE, Rosenkilde MM, Rot A, Sozzani S, Thelen M, Yoshie O, Zlotnik A. International Union of Basic and Clinical Pharmacology. [corrected]. LXXXIX. Update on the extended family of chemokine receptors and introducing a new nomenclature for atypical chemokine receptors. Pharmacological Reviews. 2013;66(1):1-79. DOI: 10.1124/pr.113.007724

[34] Kufareva I, Salanga CL, Handel TM. Chemokine and chemokine receptor structure and interactions: implications for therapeutic strategies. Immunology and Cell Biology. 2015;93(4):372-383. DOI: 10.1038/icb.2015.15

[35] Lazennec G, Richmond A. Chemokines and chemokine receptors: New insights into cancer-related inflammation. Trends in Molecular Medicine. 2010;16(3):133-144. DOI: 10.1016/j.molmed.2010.01.003

[36] Mantovani A, Savino B, Locati M, Zammataro L, Allavena P, Bonecchi R. The chemokine system in cancer biology and therapy. Cytokine \& Growth Factor Reviews. 2010;21(1):2739. DOI: 10.1016/j.cytogfr.2009.11.007

[37] Chow MT, Luster AD. Chemokines in cancer. Cancer Immunology Research. 2014;2(12): 1125-1131. DOI: 10.1158/2326-6066.CIR-14-0160

[38] Ugurel S, Schrama D, Keller G, Schadendorf D, Bröcker EB, Houben R, Zapatka M, Fink W, Kaufman HL, Becker JC. Impact of the CCR5 gene polymorphism on the survival of metastatic melanoma patients receiving immunotherapy. Cancer Immunology, Immunotherapy. 2008;57(5):685-691. DOI: 10.1007/s00262-007-0407-z

[39] Bedognetti D, Spivey TL, Zhao Y, Uccellini L, Tomei S, Dudley ME, Ascierto ML, De Giorgi V, Liu Q, Delogu LG, Sommariva M, Sertoli MR, Simon R, Wang E, Rosenberg SA, Marincola FM. CXCR3/CCR5 pathways in metastatic melanoma patients treated with adoptive therapy and interleukin-2. British Journal of Cancer. 2013;109(9):2412-2423. DOI: $10.1038 /$ bjc.2013.557

[40] Hamid O, Schmidt H, Nissan A, Ridolfi L, Aamdal S, Hansson J, Guida M, Hyams DM, Gómez H, Bastholt L, Chasalow SD, Berman D. A prospective phase II trial exploring the association between tumor microenvironment biomarkers and clinical activity of ipilimumab in advanced melanoma. Journal of Translational Medicine. 2011;9:204. DOI: $10.1186 / 1479-5876-9-204$

[41] Lanier LL. NK cell recognition. Annual Review of Immunology. 2005;23:225-274. DOI: 10.1146/annurev.immunol.23.021704.115526

[42] Venstrom JM, Zheng J, Noor N, Danis KE, Yeh AW, Cheung IY, Dupont B, O'Reilly RJ, Cheung NK, Hsu KC. KIR and HLA genotypes are associated with disease progression and survival following autologous hematopoietic stem cell transplantation for high-risk neuroblastoma. Clinical Cancer Research. 2009;15(23):7330-7334. DOI: 10.1158/1078-0432. CCR-09-1720 
[43] Delgado DC, Hank JA, Kolesar J, Lorentzen D, Gan J, Seo S, Kim K, Shusterman S, Gillies SD, Reisfeld RA, Yang R, Gadbaw B, DeSantes KB, London WB, Seeger RC, Maris JM, Sondel PM. Genotypes of NK cell KIR receptors, their ligands, and Fc $\gamma$ receptors in the response of neuroblastoma patients to Hu14.18-IL2 immunotherapy. Cancer Research. 2010;70(23):9554-9561. DOI: 10.1158/0008-5472.CAN-10-2211

[44] Paiva M, Marques H, Martins A, Ferreira P, Catarino R, Medeiros R. FcgammaRIIa polymorphism and clinical response to rituximab in non-Hodgkin lymphoma patients. Cancer Genetics and Cytogenetics. 2008;183(1):35-40. DOI: 10.1016/j.cancergencyto.2008.02.001

[45] Cartron G, Dacheux L, Salles G, Solal-Celigny P, Bardos P, Colombat P, Watier H. Therapeutic activity of humanized anti-CD20 monoclonal antibody and polymorphism in IgG Fc receptor FcgammaRIIIa gene. Blood. 2002;99(3):754-758. DOI: 10.1182/blood. V99.3.754

[46] Zhang W, Gordon M, Schultheis AM, Yang DY, Nagashima F, Azuma M, Chang HM, Borucka E, Lurje G, Sherrod AE, Iqbal S, Groshen S, Lenz HJ. FCGR2A and FCGR3A polymorphisms associated with clinical outcome of epidermal growth factor receptor expressing metastatic colorectal cancer patients treated with single-agent cetuximab. Journal of Clinical Oncology. 2007;25(24):3712-3718. DOI: 10.1200/JCO.2006.08.8021

[47] Musolino A, Naldi N, Bortesi B, Pezzuolo D, Capelletti M, Missale G, Laccabue D, Zerbini A, Camisa R, Bisagni G, Neri TM, Ardizzoni A. Immunoglobulin G fragment $\mathrm{C}$ receptor polymorphisms and clinical efficacy of trastuzumab-based therapy in patients with HER-2/neu-positive metastatic breast cancer. Journal of Clinical Oncology. 2008;26(11):1789-1796. DOI: 10.1200/JCO.2007.14.8957

[48] Cheung NK, Sowers R, Vickers AJ, Cheung IY, Kushner BH, Gorlick R. FCGR2A polymorphism is correlated with clinical outcome after immunotherapy of neuroblastoma with anti-GD2 antibody and granulocyte macrophage colony-stimulating factor. Journal of Clinical Oncology. 2006;24(18):2885-2890. DOI: 10.1200/JCO.2005.04.6011 